\title{
Narrow-band EUV multilayer coating for the MOSES sounding rocket
}

\author{
Scott M. Owens ${ }^{1 *}$, Jeffery S. Gum ${ }^{1}$, Charles Tarrio ${ }^{2}$, Steven Grantham ${ }^{2}$, Joseph Dvorak ${ }^{3}$, Benjawan \\ Kjornrattanawanich ${ }^{4}$, Ritva Keski-Kuha ${ }^{1}$, Roger J. Thomas ${ }^{1}$, Charles C. Kankelborg ${ }^{3}$ \\ ${ }^{1}$ Goddard Space Flight Center, Greenbelt, MD 20771 \\ ${ }^{2}$ National Institute of Standard and Technology, Gaithersburg, MD 20899 \\ ${ }^{3}$ Department of Physics, Montana State University, Bozeman, MT 59717 \\ ${ }^{4}$ Universities Space Research Association, National Synchrotron Light Source, Brookhaven \\ National Laboratory, Upton, NY 11973
}

\begin{abstract}
The Multi-order Solar EUV Spectrograph (MOSES) is a slitless spectrograph designed to study solar He II emission at $303.8 \AA(1 \AA=0.1 \mathrm{~nm})$, to be launched on a sounding rocket payload. One difference between MOSES and other slitless spectrographs is that the images are recorded simultaneously at three spectral orders, $m=-1,0,+1$. Another is the addition of a narrow-band multilayer coating on both the grating and the fold flat, which will reject out-of-band lines that normally contaminate the image of a slitless instrument. The primary metrics for the coating were high peak reflectivity and suppression of $\mathrm{Fe} \mathrm{XV}$ and XVI emission lines at $284 \AA$ and $335 \AA$, respectively. We chose $\mathrm{B}_{4} \mathrm{C} / \mathrm{Mg}_{2} \mathrm{Si}$ for our material combination since it provides excellent peak reflectivity and rejection of out-of-band wavelengths. Measurements of witness flats at NIST indicate the peak reflectivity at 303.8 is $39.0 \%$ for a 15 bilayer stack, while suppression ranges from $7.5 \mathrm{x}$ to $12.9 \mathrm{x}$ at $284 \AA$ and from $3.4 \mathrm{x}$ to $15.1 \mathrm{x}$ at $335 \AA$ for the individual reflections in the optical path. We present the results of coating the MOSES flight gratings and fold flat, including the spectral response of the fold flat and grating as measured at NIST's SURF III and Brookhaven's X24C beamline, respectively.
\end{abstract}

Keywords: EUV multilayers, narrow band multilayers, $\mathrm{B}_{4} \mathrm{C} / \mathrm{Mg}_{2} \mathrm{Si}$

\section{INTRODUCTION}

The solar atmosphere presents a tremendous challenge for remote sensing observations. Coronal mass ejections, flares, microflares, and other dynamic phenomena show variability at small temporal and spatial scales. The solar observer is often left with an uncomfortable compromise between spectral, spatial and temporal resolution. The desire to combine imaging and spectroscopic capabilities has produced a variety of compromises. In visible light, for example, tunable Fabry-Perot interferometers have made it possible to image slices of a spectral line profile in rapid succession. In extreme ultraviolet (EUV), the options are limited because there are no materials with high transmission coefficients. Yet this spectral range is of considerable importance to solar physics because it gives access to the sun's dynamic corona and transition region. The NRL SO82A experiment aboard Skylab ${ }^{1}$ used an objective grating to produce overlapping, dispersed images in a multitude of solar EUV spectral lines. The Multi-Order Solar EUV Spectrograph (MOSES) represents a new approach: truly simultaneous imaging and spectroscopy by recording images at several spectral orders of a narrowband objective grating (see Figure 3). Each of the images obtained by MOSES is equivalent to a projection through the three-dimensional hybrid space $(\mathrm{x}, \mathrm{y}, \lambda)$ occupied by the object. The central $(\mathrm{m}=0)$ order is a simple image. ${ }^{2}$ The two dispersed orders, like the images from SO82A, are difficult to interpret by themselves. By inversion or forward modeling, we may solve for spectra consistent with the measurements in all three spectral orders at

- $\quad$ Scott.Owens@nasa.gov

- Tel (301) 286-3197

- $\quad F a x(301) 286-0204$

Optics for EUV, X-Ray, and Gamma-Ray Astronomy II, edited by Oberto Citterio, Stephen L. O'Dell, Proc. of SPIE Vol. 5900, 590003, (2005) - 0277-786X/05/\$15 - doi: 10.1117/12.617520 
each image pixel. ${ }^{3}$ Since multilayer coatings are used on the optics, the instrument bandpass is limited to two spectral lines: He II $303.8 \AA$ and Si XI $303.3 \AA(1 \AA=0.1 \mathrm{~nm})$. Simultaneous imaging at three spectral orders, combined with a narrow bandpass, makes the spectral inversion feasible. The MOSES sounding rocket mission is a proof-of-concept for this novel technique.

In this paper, we describe the fabrication and test of multilayer coatings for the MOSES grating and fold flat. We chose $\mathrm{B}_{4} \mathrm{C} / \mathrm{Mg}_{2} \mathrm{Si}$ as the material combination due to its very high reflectivity at the target wavelength, and excellent suppression of nearby emission lines which could contaminate the image. Coating tests and flight optics coatings were done in the Optics Branch at Goddard Space Flight Center. Measurements of test and witness samples for the fold flat coatings were done at SURF III at the National Institute of Standards and Technology, and flight grating performance measurements were done at beam line X24C of the National Synchrotron Light Source at Brookhaven National Laboratory. Coatings optimized to peak at $303.6 \AA$ yield nearly $40 \%$ reflectivity and a relatively narrow $20.5 \AA$ FWHM.

\section{DEPOSITION FACILITIES}

Initial deposition tests were done in a small Commonwealth ion beam sputtering deposition chamber. ${ }^{*}$ The sputter source is a $3 \mathrm{~cm}$ ion gun, operating at $1000 \mathrm{~V}$ and $30 \mathrm{~mA}$. The base pressure of the chamber is $3 \times 10^{-7}$ Torr, and the operating pressure is $4 \times 10^{-4}$ Torr. This chamber has a computer controlled 4-target turret, with 3" diameter target capacity. The $\mathrm{B}_{4} \mathrm{C}$ and $\mathrm{Mg}_{2} \mathrm{Si}$ targets are $99.9 \%$ and $99.5 \%$ purity, respectively. Sample substrates are $50 \mathrm{~mm}$ square by $2 \mathrm{~mm}$ thick float glass, which undergo a standard aqueous cleaning process used for all test samples in our laboratory. Deposition was monitored by a Leybold quartz crystal microbalance, and controlled by software developed in-house. Samples were analyzed using grazing incidence x-ray reflectivity (GIXR) and near-normal incidence EUV reflectivity. Once the optimum recipe for the multilayer stack was verified using this small chamber, further development was moved to our 2-meter diameter chamber where the flight optics were coated.

The 2-meter chamber has been described previously, ${ }^{4}$ but in brief, houses two $16 \mathrm{~cm}$ ion beam sources directed at two fixed $30 \mathrm{~cm}$ diameter targets. Substrates are held in a rack that rotates about the center of the chamber from one sputter source to the other, and back. No other rotation stages or baffles were used during this development to enhance uniformity beyond the inherent sputtering profile, though we are in the process of testing a new rotation system now. The lateral uniformity of coating thickness was determined by GIXR measurements to be $0.055 \%$ per mm over the central $90 \mathrm{~mm}$ radius tested. The implications of this non-uniformity are shown in later in Section 4, Coating Design.

\section{METROLOGY}

Measurement of test coatings was primarily done at GSFC using a combination of grazing incidence x-ray reflectivity (GIXR) at $8.048 \mathrm{keV}$ and direct EUV measurements. The GIXR instrument consists of an Oxford Apogee Cu target Xray tube, a $\mathrm{Si}(220)$ 4-bounce channel-cut monochromator, and a $0.065 \mathrm{~mm}$ x $4 \mathrm{~mm}$ slit to filter and collimate the $\mathrm{Cu}$ $\mathrm{K} \alpha_{1} \mathrm{x}$-ray line. When operated at $45 \mathrm{kV}$ and $0.8 \mathrm{~mA}$, this system yields $20,000 \mathrm{sec}^{-1}$ in the direct beam. Samples are mounted on a pair of stacked Newport 495 rotation stages, and a Newport 850G-driven translation stage to move the sample in and out of the beam. A xenon proportional counter with a $0.2 \mathrm{~mm} \times 10 \mathrm{~mm}$ slit is used to detect the $\mathrm{x}$-rays, with a background count rate of $0.1 \mathrm{c} / \mathrm{sec}$.

Normal incidence EUV measurements are performed on a McPherson 247 spectrometer. A Penning EUV light source from Berkeley Photonics ${ }^{5}$ is mounted on the scanning slit port, and a $600 \mathrm{~mm}^{-1}$ gold-coated, grazing incidence reflection grating and $0.1 \mathrm{~mm}$ exit slit are used to pick off emission lines from the light source. In our region of interest the only lines used are from Ne II and Ne III emission. The top and bottom flanges of the sample vacuum chamber are custom rotary seals operated by hand, and have vernier graduated scales with read-out to 0.1 degree. The sample is held in a mount on the top flange that can move in and out of the beam, with a micrometer stop to repeatably position the sample

\footnotetext{
* Commercial products are mentioned only for clarity; mention of a product does not constitute endorsement by the authors or the US Government.
} 
in the beam. The detector is an Amptek MD-502 channel electron multiplier operating in ion mode mounted on the bottom flange, with the $3.8 \mathrm{~mm}$ diameter detector aperture located $50 \mathrm{~mm}$ from the sample.
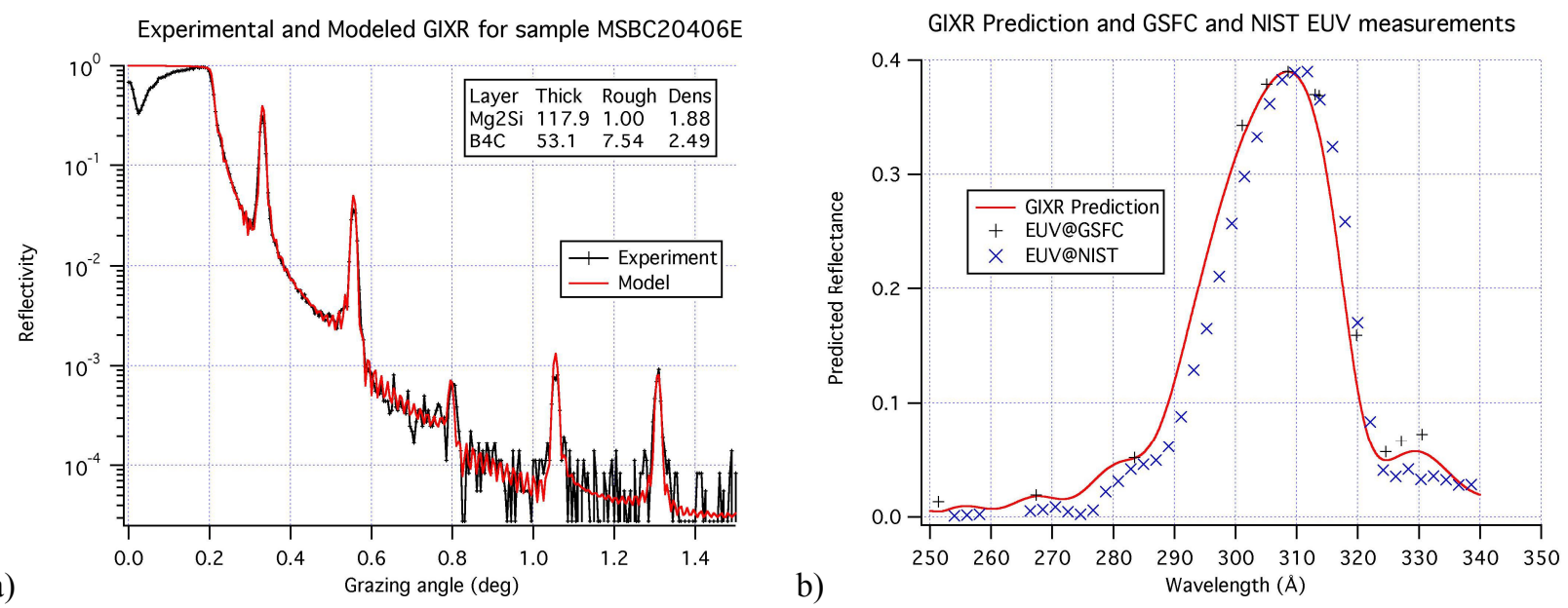

Figure 1 a) Grazing incidence x-ray reflectivity of a test mirror during the coating development process. b) Prediction and measurement of the EUV response at 5 degrees incident angle.

Using GIXR measurements, structural models of test coatings are generated, from which the EUV performance is predicted. These predictions are compared to direct EUV measurements using a gas discharge EUV light source mounted to the scanning slit of a 2.2 meter diameter McPherson reflection grating spectrometer. The absolute reflectivity obtained using this EUV reflectometer system has not been well established, so only the bandpass position information was used from these EUV measurements. Absolute EUV reflectivity measurements on important samples were performed at the NIST/DARPA EUV Reflectometry facility at NIST. ${ }^{6}$ This is a high-throughput, modest resolution instrument based on varied-line-spacing plane gratings. Uncertainties in the reflectivity values reported here are $10 \%$ of the peak value, while uncertainties in the peak wavelength are $1 \AA$.

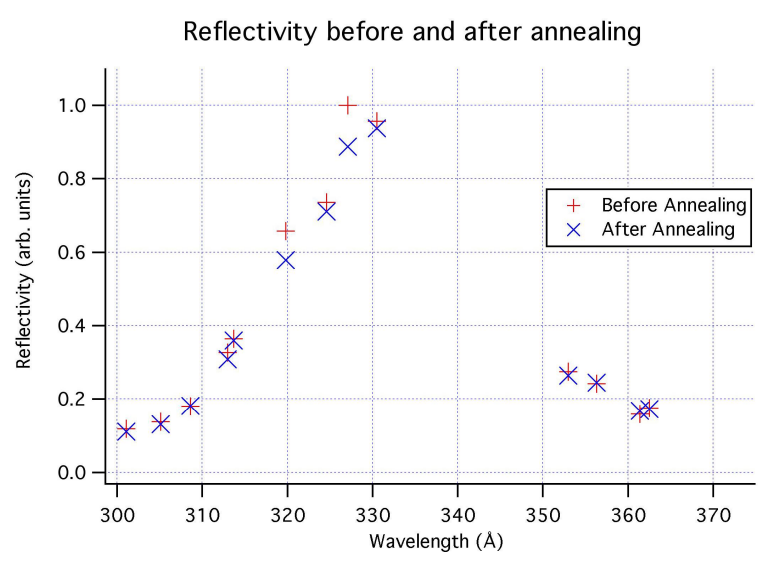

Figure 2. Annealing study of a test coating on a standard float glass slide. The sample was annealed at $100^{\circ} \mathrm{C}$ for 6 hours in ambient atmosphere.
The structural models based on GIXR measurements are found to be accurate within approximately $2 \AA$, based on comparing bandpass position predictions with NIST measurements. Peak reflectivity predictions cannot be done rigorously at this time, since the optical constants of $\mathrm{Mg}_{2} \mathrm{Si}$ are not yet well known in the $300 \AA$ region. An example of this prediction and comparison process is shown in Figure 1.

In Figure 1a), the coating is a 15 bilayer stack of $\mathrm{B}_{4} \mathrm{C} / \mathrm{Mg}_{2} \mathrm{Si}$. The model that is built and fit to the data in a) is used to predict the EUV performance by simulating the model's performance using Windt's IMD package. ${ }^{7}$ A comparison of that predicted performance and the actual

NIST measurement is shown in b). Measurements from the EUV spectrometer at GSFC are also shown for reference. The prediction curve from the IMD simulation and GSFC EUV measurements are scaled to the peak reflectivity of the NIST absolute reflectivity measurement. 
The process of tuning our deposition parameters depends critically on the use of both GIXR and normal incidence EUV reflectivity. Using just the EUV response measurements we know whether the bilayer thickness needs to be tuned up or down to place the peak at the desired position, but we do not know which layer in the period to change to maintain an optimal ratio of layer thicknesses. The most important characteristic of GIXR is that it gives us a measure of the individual thickness of the layers in a bilayer, allowing us to fully optimize the coating.

All samples passed standard tape pull tests, and showed little change upon annealing at $100^{\circ} \mathrm{C}$ for 6 hours, as shown in Figure 2.

\section{COATING DESIGN}

\section{Material considerations}

One of the primary specifications of the MOSES coatings is the rejection of out of band signal. Specifically, the Fe XV and XVI lines at $284 \AA$ and $335 \AA$ need to be suppressed as much as possible. The other primary requirement is high reflectance at $303.8 \AA$, but suppression is considered a more important factor, since the instrument is signal-to-noise limited as opposed to photon limited.

A number of candidate material combinations were considered, including $\mathrm{Si} / \mathrm{Ir}, \mathrm{Si} / \mathrm{Mo}, \mathrm{Si} / \mathrm{B}_{4} \mathrm{C}$ and a variety of materials using $\mathrm{Mg}_{2} \mathrm{Si}$ as the spacer layer. Previous experimental work ${ }^{8}$ indicated that $\mathrm{Mg}_{2} \mathrm{Si}$ may be a superior spacer layer to silicon, in that it is slightly less absorptive than silicon, and since it is also a stable compound it is less likely to produce undesirable silicides at layer interfaces. Following preliminary results from development of the SECCHI EUVI instrument, we chose to pursue further development of the $\mathrm{B}_{4} \mathrm{C} / \mathrm{Mg}_{2} \mathrm{Si}$ system, which showed high reflectivity and narrow bandpass.

Initial tests were done with 15 and 30 bilayer coatings, but simulations showed that the reflectivity of a 15 bilayer stack peaked only $1 \%$ lower than that of a 30 bilayer stack, and the FWHM of the bandpass did not appreciably change. Also, we were also concerned with the lifetime of the filaments in our ion guns, and chose to limit the risk of a filament burning out during deposition of the flight optics by proceeding with 15 bilayer coatings for both test samples and flight optics.

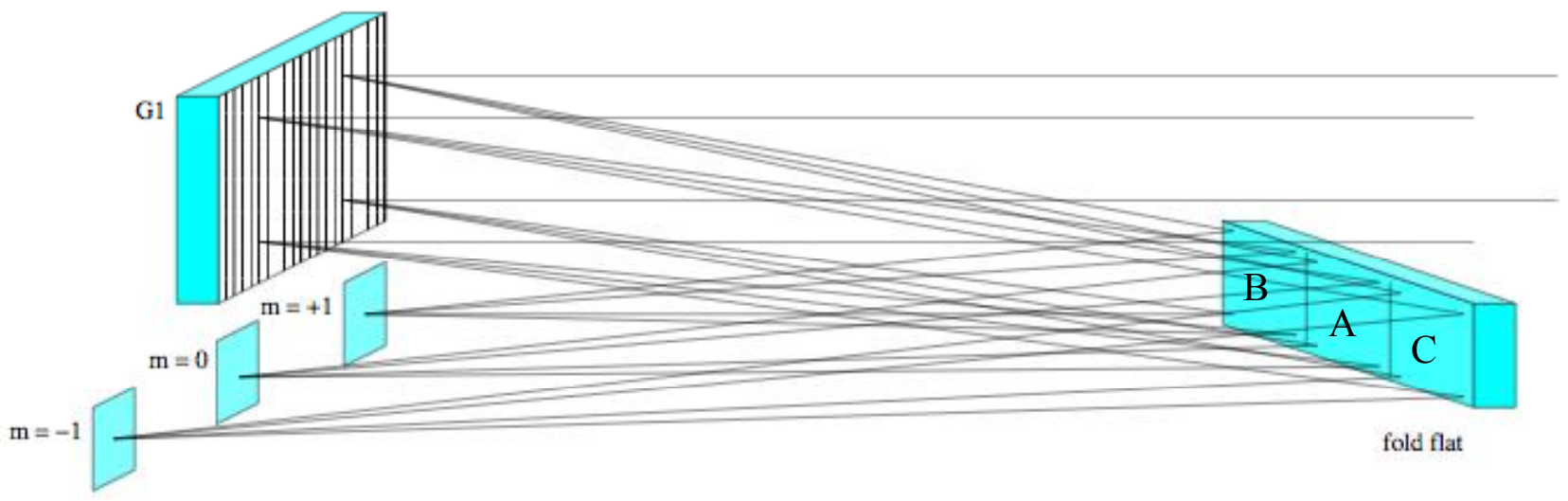

Figure 3. Schematic of the optical layout of MOSES. The spherical G1 grating reflects and disperses light onto the three sections of the fold flat, $\mathrm{A}, \mathrm{B}$ and $\mathrm{C}$, which reflects light into three imaging $\mathrm{CCD}$ detectors, corresponding to the three orders, $\mathrm{m}=0,+1$ and -1 respectively. 
a)

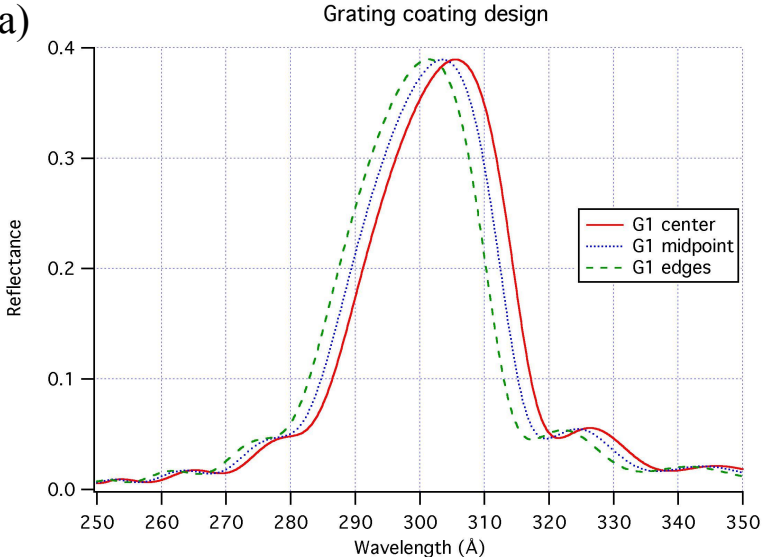

b)

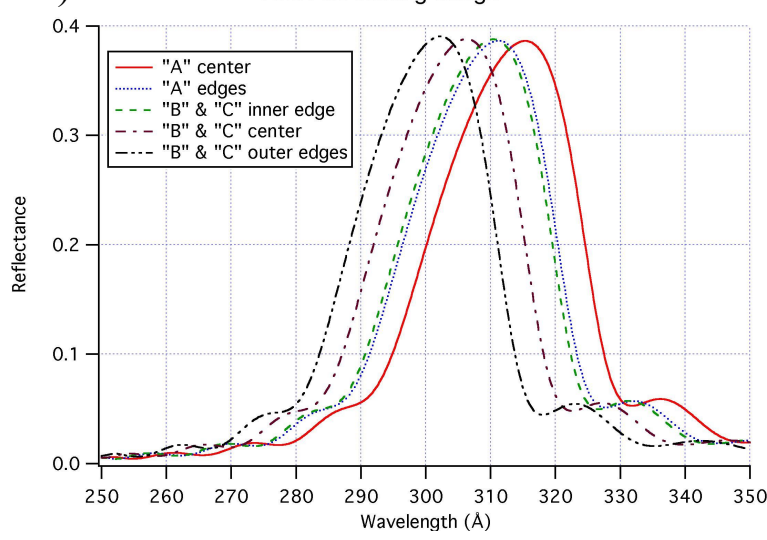

c) Reflectance of fold flat ${ }^{*}$ grating coatings

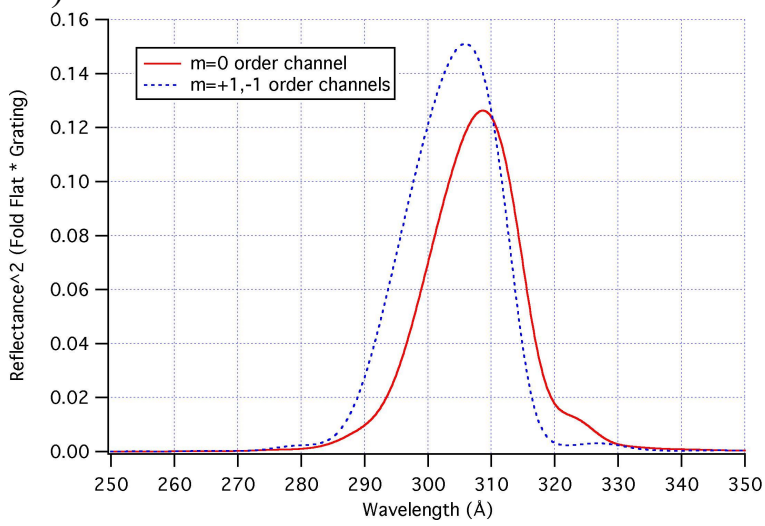

Figure 4. Grating and Fold Flat coating designs. a) EUV response of the designed coating at the center, halfway to the edges (midpoint), and at the edges of the grating. b) EUV response of the fold flat coating at a variety of positions. c) The combined EUV response of the grating and fold flat (coating only) at the center of each section.

\section{Substrates}

The grating is a $90 \mathrm{~mm}$ square, $16 \mathrm{~mm}$ thick blank, with a centered, ruled area of $80 \mathrm{~mm}$ square. The surface has a spherical curvature, with a radius of curvature of 9474 $\mathrm{mm}$, and the groove frequency is $950 \mathrm{~mm}^{-1}$. The fold flat is a rectangular mirror $193 \mathrm{~mm}$ wide by $50.8 \mathrm{~mm}$ tall by $25.4 \mathrm{~mm}$ thick, superpolished to a surface roughness of 5 $\AA$ RMS or better. The EUV reflecting surface of the fold flat was masked during coating to yield three equally sized sections, each $60 \mathrm{~mm}$ by $45 \mathrm{~mm}$. A schematic of the optical layout of MOSES is shown in Figure 3.

As can be seen in Figure $1 \mathrm{~b}$ the general shape of the reflectance curve falls more sharply on the long wavelength side, compared to the short wavelength side. Since the Fe XV $284 \AA$ line is so much closer to the center of our desired bandpass, the peak of the reflectivity curve was deliberately shifted to slightly longer wavelength in our design to optimize the balance between throughput at $303.8 \AA$ and suppression at $284 \AA$. In addition, we also needed to address the non-uniformity of the coating, especially across the surface of the fold flat.

\section{Non-uniformity issues}

As MOSES is the first multilayer program our 2-meter diameter chamber was used for, we did not have opportunity to institute rotation stages or baffling in the substrate mount to improve coating uniformity beyond the inherent distribution of the sputtering sources. As a result, very careful alignment of the substrate rack with respect to the sputter sources was required. This also meant that the d-spacing of the multilayer varies across the surface of the grating and fold flat. Even from the center of the grating to its edge, the d-spacing changes on the order of $2.5 \AA$, yielding a shift in the peak position of $4.1 \AA$. The change over the length of the fold flat is more dramatic, with a center-to-edge peak shift of $13.1 \AA$.

The variation in coating thickness along the surface leads to a choice of how to design the bandpass for optimal performance. Since the $\mathrm{m}=0$ reflection from the grating will be stronger than the $\mathrm{m}=+1$ or -1 reflections, we can afford to design the $m=+1$ and -1 sections of the fold flat (sections $\mathrm{B}$ and $\mathrm{C}$, respectively) to have approximately the same multilayer parameters as the grating. The $\mathrm{m}=0$ section of the fold flat (section A) is then shifted to longer wavelength, but the combined $\mathrm{m}=0$ channel still has a higher reflectivity than the $\mathrm{m}=+1$ and -1 order channels when the grating efficiency is taken into account. The designed bandpasses for the individual grating and fold flat, and the combined throughput are shown in Figure 4. Since we did not have an estimate of the groove efficiency 
of the grating at the time the design was done, only the effects of the multilayer coating are included in this analysis.

The combined reflectance from all combinations of positions was considered when optimizing the coating design. Since the Fe XVI line $(335 \AA)$ is further away in wavelength from He II (303.8 $\AA)$ than the Fe XV line $(284 \AA)$ we deliberately chose to bias our design toward longer wavelength. This has the benefit of balancing out the rejection of the two Fe lines and providing some insurance against any slight downward drifts in thickness during the flight mirror deposition run. The final design parameters are shown in Table 1. The values of $d$-spacing, peak position and suppression of the two Fe lines are given for the center of each optical surface.

\begin{tabular}{|l|l|}
\hline Material combination & $\mathrm{B}_{4} \mathrm{C} / \mathrm{Mg}_{2} \mathrm{Si}$ \\
\hline \# of bilayers & 15 \\
\hline d-spacing & $\mathrm{G} 1=162 \AA$ \\
& $\mathrm{FF}-\mathrm{A}=168 \AA$ \\
& $\mathrm{FF}-\mathrm{B} / \mathrm{C}=163 \AA$ \\
\hline Gamma ratio & 0.3 \\
\hline Peak position & $\mathrm{G} 1=305.5 \AA$ \\
& $\mathrm{FF}-\mathrm{A}=315.3 \AA$ \\
& $\mathrm{FF}-\mathrm{B} / \mathrm{C}=306.3 \AA$ \\
\hline $\begin{array}{l}\text { Combined } \\
\text { suppression }\end{array}$ & $\begin{array}{l}\mathrm{m}=0 \text { to } 284 \AA=39.7 \mathrm{x} \\
\mathrm{m}=0 \text { to } 335 \AA=76.9 \mathrm{x} \\
\end{array}$ \\
$\mathrm{m}=+1$ and -1 to $284 \AA=36.8 \mathrm{x}$ \\
$\mathrm{m}=+1$ and -1 to $335 \AA=240.8 \mathrm{x}$ \\
\hline
\end{tabular}

Table 1. Multilayer coating design parameters. G1 is the grating, FF-A is the central section of the fold flat (section A), and $\mathrm{FF}-\mathrm{B} / \mathrm{C}$ are the centers of the outer sections of the fold flat that reflect the $\mathrm{m}=+1$ and -1 orders from the grating

(sections B and C).

The suppression values given in Table 1 correspond to the ratio of the reflectivity of the grating times the fold flat at $303.6 \AA$ to that at $284 \AA$ and $335 \AA$ respectively. While the $240.8 \mathrm{x}$ suppression of the $335 \AA$ in the $\mathrm{m}=+1$ and -1 order channels seems unrealistically high, it is borne out in measurements of the flight coating performance.

\section{PERFORMANCE}

The EUV performance of the coated grating, G1, was measured at beamline X24C of the National Synchrotron Light Source at Brookhaven National Laboratory. The large vacuum chamber, designed for calibration of optical components and complete spaceflight and laboratory instruments, can accommodate a mirror or grating of up to $250 \mathrm{~mm}$ x $250 \mathrm{~mm}$ in size. The dual-element monochromator, comprising a gold coated mirror and a $600 \mathrm{~mm}^{-1}$ grating, provides $2.8 \mathrm{~nm}$ to $110 \mathrm{~nm}$ EUV wavelengths with spectral resolution of approximately 400. A Mg filter was used to suppress higher harmonic radiation and the $\operatorname{Mg} L$ edge was used to calibrate the wavelength scale. The spectral response was measured at the center of the grating, yielding a peak reflectivity of $15.4 \%$ at $305.3 \AA$, corresponding well with our designed peak at $305.5 \AA$. Assuming the grating coating performs as other coatings on witness samples, we estimate the groove efficiency to be $39.6 \%$. Diffraction scans at $305.3 \AA$ were measured at a variety of positions across the surface, and show $\pm 7.8 \%$ uniformity across $\mathrm{m}=0$ order peaks and proper balance between the strength of the $\mathrm{m}=+1$ and -1 orders, as shown in Figure 5. The $m=+1$ order peak is on average $47.2 \%$ of the $m=0$ order peak, while the $m=-1$ order peak is $46.7 \%$ of the $\mathrm{m}=0$ order. The spectral response is shown later in Figure 7 along with that of the fold flat. 


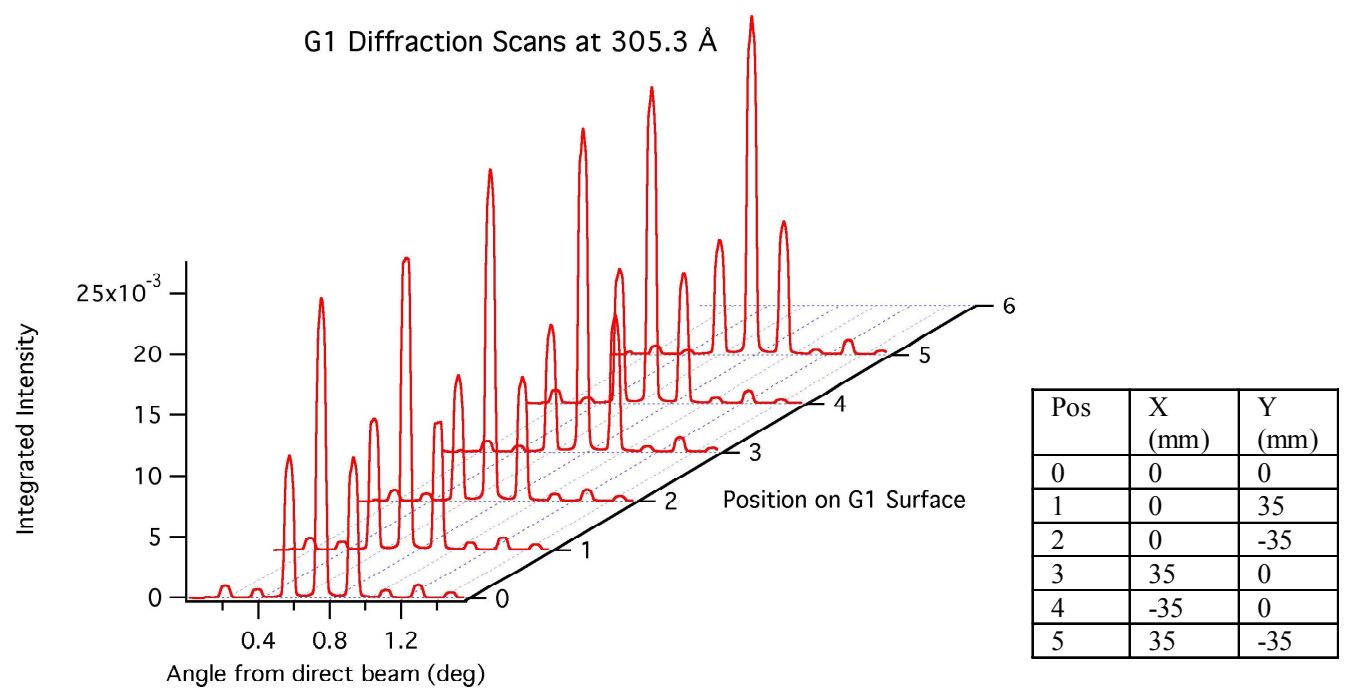

Figure 5. Diffraction scans at a variety of positions across the surface of G1. Positions indicated in the table are with respect to the center of the grating.

In order to minimize the exposure of the coated fold flat and the difficulty in handling a relatively large optic, only witness samples were measured to gauge its coated performance. For the deposition run immediately previous to coating the fold flat, six witness slides were mounted, with three placed at the center of each of the fold flat sections (positions A, B and C) and three slides were placed next to them (positions D, E and F), such that the fold flat would replace the first three in the flight coating run. (See Figure 6)

In the flight coating run, three slides were placed in the $\mathrm{D}, \mathrm{E}$ and $\mathrm{F}$ positions. These were compared to the $\mathrm{D}, \mathrm{E}$ and $\mathrm{F}$ slides from the previous run, as measured by GIXR and normal incidence EUV reflectivity at NIST. Slight shifts in the bandpass positions of the flight coating run compared to the previous run were applied to the curves from the $\mathrm{A}, \mathrm{B}$ and $\mathrm{C}$ slides from the previous run to determine the fold flat performance. These curves are shown in Figure 7a, as well as the measured performance of the coated G1 grating at $\mathrm{m}=$ 0 order.

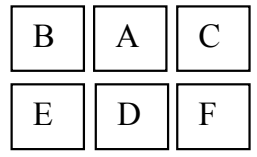

Previous coating

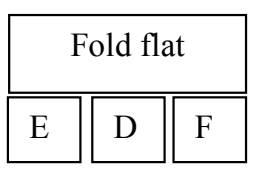

Flight coating

Figure 6. Schematic of witness slides with respect to the flight fold flat during testing and flight coating runs.

After adjusting for small shifts between deposition runs, the fold flat coating peaks in center of the A section at $315.8 \AA$, very close to our design goal of $315.3 \AA$. The $\mathrm{B}$ and $\mathrm{C}$ sections peak at $306.5 \AA$ and $308.4 \AA$, compared to the goal of $306.3 \AA$. The absolute peak reflectivity of the A, B and C reflections is $39.0 \%, 38.1 \%$ and $38.6 \%$, while the reflectivity at $303.6 \AA$ is $22.2 \%, 36.5 \%$ and $34.5 \%$, respectively. To gauge the performance of the optical system, the measured reflectivity of the G1 grating is multiplied by the measured reflectivity of fold flat witness samples, adjusted for minor shifts in the flight coating run. The result is shown in Figure $7 \mathrm{~b}$. 

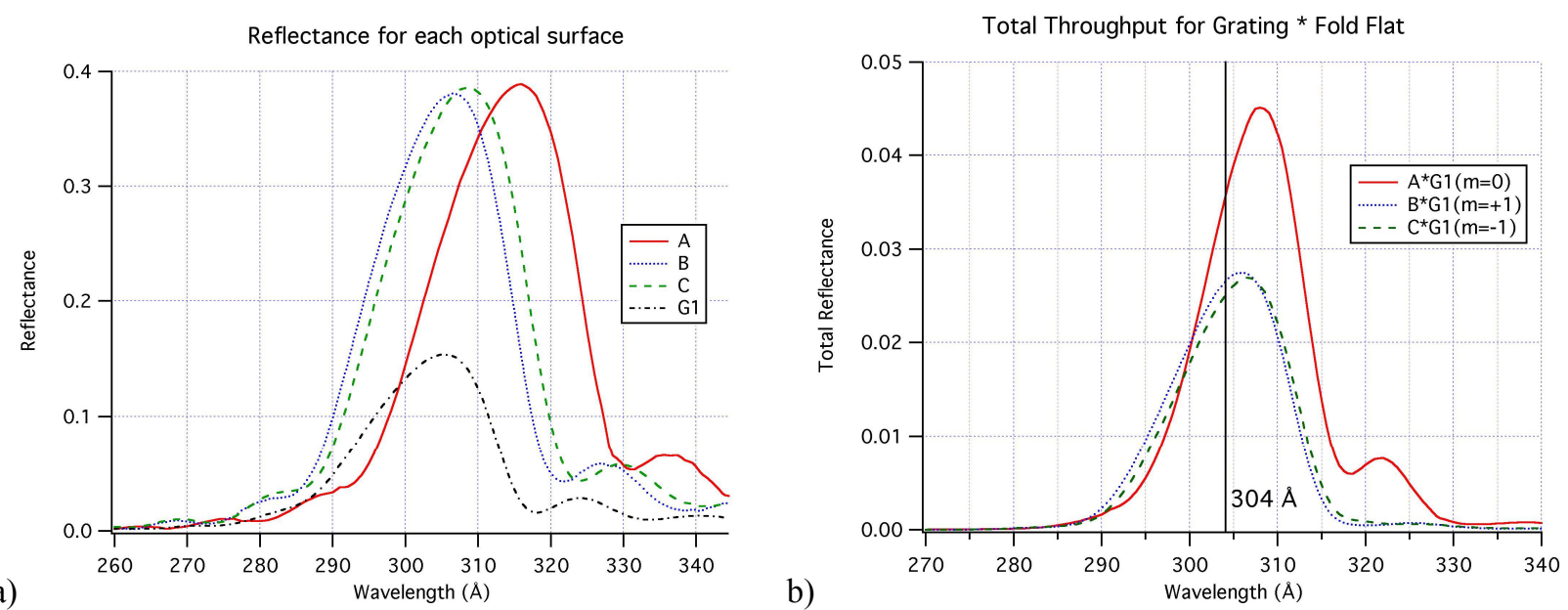

Figure 7. a) Individual reflectance of the G1 grating and fold flat. The grating curve, G1, is the reflectance at $\mathrm{m}=0$ order. b) The calculated total throughput of the optical system at the center of each channel. The relative efficiencies for the $m=+1$ and -1 orders are included, reducing the peak for these two channels.

a)

\begin{tabular}{|c|c|c|}
\hline Surface & $\begin{array}{l}\text { Reflectivity } \\
(\%)\end{array}$ & Suppression \\
\hline G1 & $\begin{array}{l}\mathrm{R}(284)=2.0 \\
\mathrm{R}(303.6)=15.1 \\
\mathrm{R}(335)=1.0\end{array}$ & $\begin{array}{l}7.5 \mathrm{x} \text { to } 284 \AA \\
15.1 \mathrm{x} \text { to } 335 \\
\AA\end{array}$ \\
\hline FF-A & $\begin{array}{l}\mathrm{R}(284)=1.6 \\
\mathrm{R}(303.6)=22.0 \\
\mathrm{R}(335)=6.5\end{array}$ & $\begin{array}{l}12.9 x \text { to } 284 \\
\AA \\
3.4 x \text { to } 335 \AA\end{array}$ \\
\hline FF-B & $\begin{array}{l}\mathrm{R}(284)=3.0 \\
\mathrm{R}(303.6)=36.5 \\
\mathrm{R}(335)=2.5\end{array}$ & $\begin{array}{l}12.1 \mathrm{x} \text { to } 284 \\
\AA \\
14.5 \mathrm{x} \text { to } 335 \\
\AA\end{array}$ \\
\hline FF-C & $\begin{array}{l}\mathrm{R}(284)=3.4 \\
\mathrm{R}(303.6)=34.5 \\
\mathrm{R}(335)=3.7\end{array}$ & $\begin{array}{l}10.1 \mathrm{x} \text { to } 284 \\
\AA \\
9.5 \mathrm{x} \text { to } 335 \AA\end{array}$ \\
\hline
\end{tabular}

b)

\begin{tabular}{|l|l|l|}
\hline Order & Reflectivity (\%) & Suppression $(\mathrm{x})$ \\
\hline $\mathrm{m}=0$ & $\mathrm{R}(284)=0.0338$ & $98.6 \mathrm{x}$ to $284 \AA$ \\
& $\mathrm{R}(303.6)=3.33$ & $51.0 \mathrm{x}$ to $335 \AA$ \\
& $\mathrm{R}(335)=0.0653$ & \\
\hline $\mathrm{m}=+1$ & $\mathrm{R}(284)=0.0280$ & $92.7 \mathrm{x}$ to $284 \AA$ \\
& $\mathrm{R}(303.6)=2.59$ & $223.4 \mathrm{x}$ to $335 \AA$ \\
& $\mathrm{R}(335)=0.0116$ & \\
\hline $\mathrm{m}=-1$ & $\mathrm{R}(284)=0.0317$ & $76.4 \mathrm{x}$ to $284 \AA$ \\
& $\mathrm{R}(303.6)=2.42$ & $137.7 \mathrm{x}$ to $335 \AA$ \\
& $\mathrm{R}(335)=0.0176$ & \\
\hline
\end{tabular}

Table 2. Suppression of Fe XV and XVI lines for a) individual reflections, and b) the two-reflection system. Values include the groove efficiencies for $\mathrm{m}=+1$ and -1 order reflections for the grating.

From this last result, we can determine the ultimate performance of the coatings, which is the suppression of the Fe XV and XVI lines with respect to the $303.6 \AA \mathrm{He}$ II line, for individual reflections and the two-reflection system. Again, suppression is determined by taking the ratio between the reflectivity at $303.6 \AA$ and that at $284 \AA$ and $335 \AA$.

For individual reflections, where the bandpass peaks near $304 \AA$, suppression of nearby Fe lines is approximately a factor of 11. For telescope systems with two reflections, this means an improvement of a factor of nearly 100 can be achieved over more conventional broadband coatings. In terms of suppression, $\mathrm{B}_{4} \mathrm{C} / \mathrm{Mg}_{2} \mathrm{Si}$ performs as well as any material combination reported to date, with the possible exception of $\mathrm{Si} / \mathrm{SiC}{ }^{9}$ The very high peak reflectivity of $39 \%$ is also impressive, being a third higher than $\mathrm{Si} / \mathrm{B}_{4} \mathrm{C}$. Suppression of nearby lines ranges from 51 times to 223 times for the combined system, shown in Table 2. 


\section{SUMMARY}

The Multi-order Solar EUV Spectrograph (MOSES) is a slitless spectrometer designed to observe the sun at the $303.6 \AA$ He II line. In order to simplify data analysis, the instrument requires a narrow band reflective coating to reject photons from nearby emission lines that will contaminate the images with undesired wavelengths. We have developed and demonstrated the performance of the $\mathrm{B}_{4} \mathrm{C} / \mathrm{Mg}_{2} \mathrm{Si}$ multilayer combination for this purpose. The characteristics of nearly $40 \%$ reflectivity at the He II line, a narrow FWHM of 20 Angstroms, and good thermal and adhesion performance makes this material combination ripe for further development and consideration on long-term solar observing missions.

\section{ACKNOWLEDGEMENTS}

The authors would like to acknowledge support from NASA grant NAG5-10997, which funds the MOSES sounding rocket mission. We would also like to thank Carl Zeiss Laser Optics for supplying the grating and working hard to ensure it met the MOSES specifications.

\footnotetext{
${ }^{1}$ Tousey, R., Bartoe, J.-D.F., Brueckner, G.E., and Purcell, J.D., "Extreme Ultraviolet Spectroheliograph ATM Experiment SO82A," Appl. Optics, 16, 870 (1977).

${ }^{2}$ Kankelborg, C.C., and Thomas, R.J., "Simultaneous Imaging and Spectroscopy of the Solar Atmosphere: Advantages and Challenges of a 3-Order Slitless Spectrograph," Proc. SPIE, 4498, 16 (2001).

${ }^{3}$ Fox, J.L., Kankelborg, C.C., and Metcalf, T.R., "Data Inversion for the Multi-Order Solar Extreme-Ultraviolet Spectrograph," Proc. SPIE, 5157, 124 (2003).

${ }^{4}$ R. A. M. Keski-Kuha, J. F. Osantowski, H. Herzig, J. S. Gum, and A. R. Toft, "Normal incidence reflectance of ion beam deposited $\mathrm{SiC}$ films in the EUV," Appl. Opt.27, 2815 (1988).

5 D. S. Finley, S. Bowyer, F. Paresce and R. F. Malina, "Continuous discharge Penning source with emission lines between $50 \AA$ and $300 \AA$ Å,” Appl. Opt., v 18, p 649 (1979).

6 "Facility for extreme ultraviolet reflectometry of lithography optics," C. Tarrio, S. Grantham, T. B. Lucatorto, Metrologia 40, S229 (2003).

${ }^{7}$ D. L. Windt, "Computers in Physics", 12, 360 (1998).

${ }^{8}$ M. F. Ravet, et. al, unpublished results.

${ }^{9}$ D. L. Windt, S. Donguy, J. Seely, B. Kjornrattanawanich, E. M. Gullikson, C. C. Walton, L. Golub, E. DeLuca "EUV Multilayers for Solar Physics", Proc. SPIE, 5168, 01 (2003).
} 